\title{
THE DIFFUSION OF A FLUID THROUGH A HIGHLY ELASTIC SPHERICAL MEMBRANE
}

\author{
K. R. RAJAGOPAL \\ Department of Mechanical Engineering, University of Pittsburgh, Pittsburgh, PA 15261, U.S.A. \\ A. S. WINEMAN \\ Department of Mechaniclal Engineering and Applied Mechanics, The University of Michigan, Ann Arbor, \\ MI $43: 09$, U.S.A. \\ and \\ JOHN JIN-JAU SHI \\ Rocket Dyne, Division of Rockwell International, Kenoga Park, California, U.S.A.
}

\section{INTRODUCTION}

A MATHEMATICAL theory of elastic materials valid for membrane like bodies has been presented in the treatise on non-linear elasticity by Green and Adkins [1]. This theory has been used extensively in solving specific boundary value problems. The same theory has also been employed to solve problems involving membranes of viscoelastic materials [2]. This theory is essentially two dimensional in that the governing equations, which hold on the middle surface in the limit of vanishing thickness, are assumed to hold even when the ratio of the thickness to a typical dimension is small but finite. This implies that the variation of the stretch ratios and related quantities are negligible along the thickness and are hence taken to be constant.

There are several problems of practical interest of which the problem of filtration is one where a membrane theory for diffusion would be appropriate. It is our aim to develop such a membrane theory which would be applicable in the case of interacting continua. The membrane theory as developed by Green and Adkins [1] for elastic solids forms the basis for our work. It is, however, found that modifications have to be made to the theory if it is to be compatible with the theory of interacting continua.

The problem of the diffusion of an ideal fluid through a spherical shell of non-linearly elastic material has been previously studied [3,4]. It was found that the stretch ratios were not constant in the radial direction. The gradient of the stretch ratio in the radial direction though small, was not negligible (about 10\%). These gradients in the stretch ratios would be present even if the thickness of the shell were to become very small, i.e. in the membrane approximation. This new feature is a consequence of the presence of diffusive body forces which depend on the gradients of the densities and stretch tensors. Hence, in this work, we develop a membrane theory which depends on the values at the deformed middle surface of physical quantities and their gradients in the thickness direction. In order to avoid the question of flow within the membrane middle surface due to gradients in this surface, we confine attention to the axially symmetric problem of the diffusion of an ideal fluid through a spherical membrane of a nonlinear elastic material.

After a brief review of the notations and basic equations relevant to a mixture of interacting continua in Section 2, we introduce a specific constitutive relation which is useful in describing the behavior of rubber-like nonlinearly elastic solids in Section 3. The forms of the constitutive relations employed are obtained by incorporating expressions suggested in the kinetic theory of rubber elasticity ( $c f$. Treloar [5]) for the specific internal energy function. The phenomenon of swelling is introduced in Section 4. The problem inherent to specifying the traction boundary condition for interacting continua is overcome by assuming that the swollen state of the mixture is a saturated state. This permits the use of a relation between the surface tractions and the amount of stretching in the saturated state and helps resolve the problem of specifying the boundary condition. The kinematical quantities and the associated geometric relations pertinent to the deformed state are developed in Section 5. The equilibrium equations for the two constituents and the membrane approximations are developed in Section 6. The 
complete system of equations needed for the solution of the problem of diffusion through a spherical membrane, applicable for a large class of rubber-like materials [5], is presented in Sections 7 and 8 . The method of solution is discussed in Section 9, and a specific problem is solved in Section 10.

\section{PRELIMINARIES}

For the sake of brevity, we shall refrain from a detailed discussion of the basic balance laws, the thermodynamic considerations and their consequence on the constitutive structure of the mixture. We refer the reader to [4] for details.

A mixture of two continua, which are in motion relative to each other, is considered. The relative motion is caused by a fluid $S_{2}$ diffusing through a solid $S_{1}$. Let $\mathbf{x}=\chi_{\mathbf{1}}(\mathbf{X}, \mathbf{t})$ and $\mathbf{y}=\chi_{2}(Y, t)$ denote the motion of the solid and fluid, respectively. Let $\mathbf{u}, \mathbf{f}$ and $\mathbf{v}, \mathbf{g}$ denote the velocity and acceleration vectors of $S_{1}$ and $S_{2}$, respectively.

The deformation gradient tensor for the solids $S_{1}$ is $\mathbf{F}=\partial \boldsymbol{x}_{1} / \partial \mathbf{X}$. The densities of $S_{1}$ and $S_{2}$ at time $t$, measured per unit volume of mixture, are denoted by $\rho_{1}$ and $\rho_{2}$, respectively. The mean velocity of the mixture and the total density of the mixture are then defined by

$$
\rho \mathbf{w}=\rho_{1} \mathbf{u}+\rho_{2} \mathbf{v}, \rho=\rho_{1}+\rho_{2} .
$$

The appropriate form of the mass balance equation for the solid and fluid are

$$
\rho_{1} \operatorname{det} \mathbf{F}=\rho_{10} \text { and } \frac{\partial \rho_{2}}{\partial t}+\operatorname{div}\left(\rho_{2} \mathbf{v}\right)=0
$$

where $\rho_{10}$ is the mass density of the solid before forming the mixture.

Let $\sigma$ and $\pi$ be the partial stress tensors for $S_{1}$ and $S_{2}$, respectively. Let b denote the diffusive body force. In the absence of external body force, the equations of motion for $S_{1}$ and $S_{2}$ are

$$
\operatorname{div} \boldsymbol{\sigma}-\mathbf{b}=\rho_{1} f, \quad \operatorname{div} \boldsymbol{\pi}+\mathbf{b}=\rho_{2} \mathbf{g},
$$

and

$$
\boldsymbol{\sigma}+\boldsymbol{\pi}=\boldsymbol{\sigma}^{T}+\boldsymbol{\pi}^{T}
$$

For the sake of completeness, note that by setting $\mathbf{b}=\operatorname{grad} \phi+\overline{\mathbf{b}}, \boldsymbol{\sigma}=\phi \mathbf{1}+\bar{\sigma}, \pi=-\phi \mathbf{1}+\overline{\boldsymbol{\pi}}$, the form of eqn (3) in terms of $(\overline{\mathbf{b}}, \overline{\boldsymbol{\sigma}}, \overline{\boldsymbol{\pi}})$ is the same as in terms of $(\mathbf{b}, \boldsymbol{\sigma}, \boldsymbol{\pi})$. The scalar $\phi$ was introduced in [6] to simplify certain thermodynamic arguments. It plays no role here and will not be mentioned further. The remainder of the paper is concerned with $(\overline{\mathbf{b}}, \overline{\boldsymbol{\sigma}}, \overline{\boldsymbol{n}})$. For notational convenience, the bar will be dropped.

We assume that $S_{1}$ is an incompressible elastic solid and $S_{2}$ is an incompressible ideal fluid. In their individual reference configurations, let $S_{1}$ and $S_{2}$ have constant densities $\rho_{10}$ and $\rho_{20}$ and volumes $V_{1}$ and $V_{2}$, respectively. We shall assume that the volume of the mixture is $V_{1}+V_{2}$. It can be shown that the current densities satisfy the following equation ( $c f$. Mills [7]):

$$
\frac{\rho_{1}}{\rho_{10}}+\frac{\rho_{2}}{\rho_{20}}=1
$$

Constitutive assumptions are presented in [4] for $\overline{\mathbf{b}}, \overline{\boldsymbol{\sigma}}$ and $\overline{\boldsymbol{\pi}}$ in terms of a mean specific Helmholtz free energy for the mixture $A$. We assume the following form for $A$ :

$$
A=K\left[I_{1}-3+\ln \left(1-\left(\rho_{2} / \rho_{20}\right)\right)\right],
$$

where $K=R T / 2 M_{c}$ and $I_{1}=\operatorname{tr} \mathbf{C}, \mathbf{C}=F^{T}$. Also, $T$ is the absolute temperature, $R$ is the gas constant and $M_{c}$ is the molecular weight between cross-links. It follows that

$$
\sigma_{\mathrm{ki}}=-p \frac{\rho_{1}}{\rho_{10}} \delta_{i k}+2 \rho K C_{k} \text {, }
$$




$$
\begin{gathered}
\pi_{k i}=-p \frac{\rho_{2}}{\rho_{20}} \delta_{k i}+\rho \frac{\rho_{2}}{\rho_{20}} \frac{\rho_{10}}{\rho_{1}} K \delta_{k i}, \\
b_{k}=-\frac{p}{\rho_{10}} \frac{\partial \rho_{1}}{\partial x_{k}}-K \frac{\rho_{10}}{\rho_{20}} \frac{\partial \rho_{2}}{\partial x_{k}}-\rho_{2} K \frac{\partial I_{1}}{\partial x_{k}}+\alpha \frac{\rho_{1}}{\rho_{10}} \frac{\rho_{2}}{\rho_{20}}\left(u_{k}-v_{k}\right) .
\end{gathered}
$$

\section{SWELLING AND SATURATION}

When a rubber-like nonlinearly elastic material is immersed in certain fluids (solvents), the rubber absorbs the fluid and undergoes dimensional changes. If the rubber is pre-strained and then immersed in the fluid, the amount of the fluid absorbed is generally different than in the unstrained case. In general, if sufficient amount of fluid is present, this absorption process carries on to the state where no further absorption is possible, that is, the mixture is saturated. Treloar [5] observes that "For an unvulcanized rubber in a good solvent the swelling may proceed to an unlimited extent, the rubber and the liquid being miscible or mutually soluble in all proportions." In this analysis we shall restrict ourselves to mixtures with saturated and limited swollen states.

Adkins [8] was the first to consider the problem of swelling using particular constitutive relations within the framework of the theory of mixtues. He considered an isotropic elastic cube surrounded by an ideal fluid undergoing uniform swelling. He did not consider problems which incorporated the constraint expressed by eqn (4) of our analysis. Also, he assumed that the surfaces of the mixture were free of traction. Two equations relating the stretch ratio of each side of the swollen cube, the density $\rho_{1}$ of the solid and density $\rho_{2}$ of the fluid can be obtained from the expressions for total stress and conservation of mass for the solid. Under these conditions, it is not possible to uniquely determine the swollen condition. However, if the swollen state is a saturated state we can supplement the above equations with an additional condition based on thermodynamic equilibrium arguments which renders the problem determinate. This additional condition is the well known Flory-Huggins equation ( $c f$. Treloar [5]) which for a cube of material has the form,

$$
\left(\frac{t^{11}+t^{22}+t^{33}}{3}+p_{s}\right) \frac{V_{1}}{R T}=\ln (1-\nu)+\nu+\chi \nu^{2}+\frac{\rho_{10} V_{1}}{M_{c}}\left[\frac{\nu}{3}\left(\lambda_{1}^{2}+\lambda_{1}^{2}+\lambda_{3}^{2}\right)-\frac{\nu}{2}\right]
$$

where $\lambda_{1}, \lambda_{2}, \lambda_{3}$ are the stretch ratios of the edges of the cube, $\nu=I_{3}^{-1 / 2}=\left(\lambda_{1} \lambda_{2} \lambda_{3}\right)^{-1}$ is the volume fraction of the solid constituent and $t^{i j}=\sigma^{i j}+\pi^{i j}$ is the total stress. $R, T$ and $M_{\mathrm{c}}$ are defined in Section 3, $V_{1}$ in the molar volume of the fluid, $p_{s}$ is the hydrostatic pressure of the surrounding fluid, and $\chi$ is a constant depending on the particular rubber-fluid mixture.

It is needless to go into a detailed derivation of eqn (8) at this juncture. However, it is important to emphasize here the departure from its usual application, namely its being employed as a boundary condition. The details regarding the departure in deploying eqn (8) as a boundary condition and the rationale for the same are provided in Section 8.

\section{GEOMETRICAL RELATIONS FOR THE DEFORMED MEMBRANE}

The undeformed state of the solid constituent is a hollow spherical shell of thickness $h_{0}$ which is assumed to be very small in comparison to the inner radius $R_{i}$. In the deformed state the region occupied by the mixture coincides with the region occupied by the swollen solid constituent. We shall assume that the thickness $h$ of the solid constituent in the swollen state is small in comparison to the original radius so that the deformed configuration is yet essentially a membrane. $\dagger$

We refer the description of the problem to a spherical polar coordinate system. Let $M$ denote the deformed middle surface of the fluid filled membrane and let $\overline{\mathbf{r}}(\theta, \phi)$ be the position of the point at $(\theta, \phi)$ on $M$. A particle originally at $(R, \theta, \phi)$ is now at $(r(R), \theta, \phi)$. It is a consequence of the symmetry of the problem that the $(r, \theta, \phi)$ directions are all principal directions of stress and stretch.

tWhile one might start with a body which qualifies to be a membrane, in view of Treloar's remarks on unrestrained swelling, it is possible that one might end up with a swollen body which cannot be considered as membrane. Of course, we shall preclude such an occurrence. 
Let $y_{3}$ denote the distance measured in the normal direction from the middle surface of the deformed membrane. The position vector of any point within the mixture is then defined through

$$
\mathbf{r}=\overline{\mathbf{r}}(\theta, \phi)+y_{3} \mathbf{e}_{r}
$$

and

$$
r=a+y_{3}
$$

where $a$ denotes the radius of the mid-surface in the deformed state. Let $\overline{\mathbf{R}}(\theta, \phi)$ denote the position of a particle on the surface in the undeformed state at $(\theta, \phi)$ which corresponds to the middle surface in the deformed state. Then the position of a particle of the membrane in the undeformed state can be expressed as

$$
\mathbf{R}=\overline{\mathbf{R}}(\theta, \phi)+f\left(y_{3}\right) \mathbf{e}_{r}
$$

and

$$
R=A+f\left(y_{3}\right)
$$

where $A$ denotes the radius of the surface in the undeformed state which corresponds to the middle surface in the deformed state.

The principal stretch ratios given by $\lambda_{r}, \lambda_{\theta}$ and $\lambda_{\phi}$ are defined by

$$
\lambda_{r}=\frac{\mathrm{d} r}{\mathrm{~d} R}, \lambda_{\theta}=\lambda_{\phi}=\frac{r}{R}
$$

For reasons mentioned in the introduction, we shall express all quantities in the form of a Taylor series about $r=a$, and retain the linear terms.

When $y_{3}=0, r=a$ and $R=A$, hence

$$
f(0)=0 \text {. }
$$

Thus, one obtains that

$$
f\left(y_{3}\right)=c y_{3}+0\left(y_{3}^{2}\right)
$$

where

$$
c \equiv \frac{\mathrm{d} f}{\mathrm{~d} y_{3}}(0)
$$

Substituting eqns (10) and (11) into the expressions for $\lambda_{\theta}$ in eqn (12), and then using the expansion in eqn (14), we obtain

$$
\begin{aligned}
\lambda_{\theta} & =\frac{a}{A}\left(1+\frac{y_{3}}{a}\right)\left(1+\frac{c}{A} y_{3}+O\left(y_{3}^{2}\right)\right)^{-1}=\frac{a}{A}\left[1+\left(\frac{1}{a}-\frac{c}{A}\right) y_{3}\right]+O\left(y_{3}^{2}\right) \\
& =\lambda_{m}+\lambda_{*} y_{3}+O\left(y_{3}^{2}\right)
\end{aligned}
$$

Following a similar procedure for $\lambda_{r}$, we obtain

$$
\lambda_{r}=\frac{1}{c}+c_{*} y_{3}+O\left(y_{3}^{2}\right)
$$

where

$$
c_{*}=-\frac{1}{c^{2}} \frac{d^{2} f}{d y_{3}^{2}}(0)
$$


Note that $\lambda_{*}$ can be expressed in terms of $\lambda_{m}=a / A$ and $c$. It also follows that

$$
\frac{h_{0}}{h}=c+O(h)
$$

It will be convenient at this point to develop analogous expressions for the densities. Since the $(r, \theta, \phi)$ directions are principal directions, the mass balance equation for the solid constituent is

$$
\frac{\rho_{1}}{\rho_{10}} \lambda_{r} \lambda_{\theta}^{2}=1
$$

It follows that the densities are defined by

$$
\begin{gathered}
\frac{\rho_{\beta}}{\rho_{\beta 0}}=\rho_{\beta m}+\rho_{\beta *} y_{3}+O\left(y_{3}^{2}\right), \beta=1,2 \\
\frac{\rho}{\rho_{10}}=\rho_{m}+\rho_{*} y_{3}+O\left(y_{3}^{2}\right) .
\end{gathered}
$$

It now follows from eqns (18) and (19), that

$$
\rho_{1 m} \lambda_{m}^{2}=c,
$$

and

$$
\rho_{1 *} \lambda_{m}^{2}+2 \lambda_{m} \lambda_{*} \rho_{1 m}+\rho_{1 m} \lambda_{m}^{2} c c_{*}=0
$$

From the volume additivity constraint condition (4),

$$
\rho_{1 m}+\rho_{2 m}=1, \rho_{1 *}+\rho_{2 *}=0 .
$$

From $(1)_{2}$, and $(19)_{1}-(19)_{3}$, it follows that

$$
\begin{aligned}
& \rho_{m}=\rho_{1 m}+\frac{\rho_{20}}{\rho_{10}} \rho_{2 m}, \\
& \rho_{*}=\rho_{1 *}+\frac{\rho_{20}}{\rho_{10}} \rho_{2 *} .
\end{aligned}
$$

For the present problem, eqn (2) $)_{2}$, which expresses the conservation of mass for the fluid constituents, reduces to

$$
r^{2} \rho_{2} V=F
$$

where $V$ is the radial velocity component for the fluid and $F$ is the mass flux, a constant. Writing

$$
V=V_{m}+V_{*} y_{3}+0\left(y_{3}^{2}\right),
$$

eqns (19), and (20) give that

$$
a^{2} \rho_{2 m} V_{m}=F / \rho_{20} \text { and } 2 a \rho_{2 m} V_{m}+a^{2} \rho_{2 m} V_{*}+a^{2} \rho_{2 *} V_{m}=0
$$

\section{EQUILIBRIUM}

In the example to be considered, the solid constituent is at rest. Since the flow of the fluid is assumed to be slow, we shall neglect the inertial terms occurring in the equations of motion for the fluid constituent. Let $\sigma^{i j}, \pi^{i j}$ and $b^{i}$ denote the physical components of the partial stresses 
and diffusive body force with respect to spherical coordinates. Because of the symmetry of the problem, the only equations that need to be considered are

$$
\frac{\mathrm{d} \sigma^{11}}{\mathrm{~d} r}+\frac{2\left(\sigma^{11}-\sigma^{22}\right)}{r}-b=0, \frac{\mathrm{d} \pi^{11}}{\mathrm{~d} r}+\frac{2\left(\pi^{11}-\pi^{22}\right)}{r}+b=0
$$

where $b=b^{1}$. Since eqns $(26)_{1.2}$ hold at each point in the deformed domain, they can be added to give an equation involving the total stress $t^{i j}=\sigma^{i j}+\pi^{i i}$, namely,

$$
\frac{\mathrm{d} t^{11}}{\mathrm{~d} r}+\frac{2\left(t^{11}-t^{22}\right)}{r}=0
$$

The partial stress $\sigma^{\prime \prime}(r)$ which acts normal to the radial surface at radius $r$ is measured per unit area of this surface. Let $\hat{\sigma}^{11}(r)$ denote a partial stress acting normal to the radial surface $r$, but which is measured per unit area of the deformed middle surface of radius $a$. It is defined by the relation

$$
\hat{\sigma}^{\prime \prime}(r)=\sigma^{11}(r) \frac{r^{2}}{a^{2}}
$$

On integrating the first of eqns (26) in the thickness direction, one obtains

$$
a^{2}\left[\hat{\sigma}^{\prime \prime}\left(r_{0}\right)-\hat{\sigma}^{11}\left(r_{i}\right)\right]=\int_{r_{i}}^{r_{\prime \prime}} 2 r \sigma^{22} \mathrm{~d} r+\int_{r_{i}}^{r_{0}} r^{2} b \mathrm{~d} r
$$

where, by eqn (10), $r_{0}=a+h / 2, r_{i}=a-h / 2$. We also write

$$
\hat{\sigma}^{11}(r)=\hat{\sigma}_{m}^{11}+\hat{\sigma}_{*}^{11} y_{3}+O\left(y_{3}^{2}\right)
$$

where

$$
\hat{\sigma}_{m}^{\prime \prime}=\hat{\sigma}^{\prime \prime}(a), \hat{\sigma}_{*}^{\prime \prime}=\frac{\mathrm{d} \hat{\sigma}^{\prime \prime}}{\mathrm{d} r}(a) .
$$

On substituting eqns (30) $)_{1.2}$ into eqn (29), one obtains that

$$
a^{2} \hat{\sigma}_{*}^{11}+O(h)=\frac{1}{h} \int_{-h / 2}^{h / 2} 2\left(a+y_{3}\right) \sigma^{22} \mathrm{~d} y_{3}+\frac{1}{h} \int_{-h / 2}^{h / 2}\left(a+y_{3}\right)^{2} b \mathrm{~d} y_{3} .
$$

In the limit as $h \rightarrow 0$, eqn (31) becomes

$$
a \hat{\sigma}_{*}^{11}=2 \sigma_{m}^{22}+a b_{m},
$$

where the subscript $m$ denotes that a quantity is to be evaluated at the mid surface. Similarly, we obtain for the fluid constituent

$$
a \hat{\pi}_{*}^{11}=2 \pi_{m}^{22}-a b_{m}
$$

Finally, if $p_{1}$ and $p_{2}$ are the pressures at $r_{i}$ and $r_{0}$, respectively, measured per unit area of the midsurface, then

$$
t^{11}\left(r_{0}\right)=-p_{2} \frac{a^{2}}{r_{0}^{2}}, t^{11}\left(r_{i}\right)--p_{1} \frac{a^{2}}{r_{i}^{2}}
$$

The corresponding equation for the total stress in the limit $h \rightarrow 0$ is

$$
\frac{a}{h}\left(p_{1}-p_{2}\right)=2 t_{m}^{22}
$$


In the previous section the equilibrium equations for the membrane approximation were developed in the general case. We now specialize the constitutive equations presented in Section 3 for use with these equations. In particular, we first obtain the equations in spherical coordinates. Next, we eliminate the indeterminate scalar $p$ and hence simplify the equations. Finally, we express the resulting relations in terms of the quantities defined on the deformed middle surface.

For the current example, the only non-zero components of the stretch tensor $\mathbf{C}$ are $C_{11}=\lambda_{r}^{2}$, $C_{22}=C_{33}=\lambda_{\theta}^{2}$. The invariants of $\mathbf{C}$ are

$$
I_{1}=\lambda_{r}^{2}+2 \lambda_{\theta}^{2}, I_{2}=2 \lambda_{r}^{2} \lambda_{\theta}^{2}+\lambda_{\theta}^{4} \text { and } I_{3}=\lambda_{r}^{2} \lambda_{\theta}^{4} .
$$

The physical components of the partial stresses with respect to the spherical coordinates are obtained from eqn (6). These are as follows:

$$
\begin{gathered}
\sigma^{i i}=-p \frac{\rho_{1}}{\rho_{10}}+S^{i i}, \pi^{i i}=-p \frac{\rho_{2}}{\rho_{20}}+\pi^{i i}, \text { no sum on } \\
\sigma^{i j}=\pi^{i j}=0, i \neq j . \\
S^{11}=2 \rho K \lambda_{r}^{2}, S^{22}=S^{33}=2 \rho K \lambda_{\theta}^{2} \\
\pi^{11}=\pi^{22}=\pi^{33}=\rho K \frac{\rho_{2}}{\rho_{1}} \frac{\rho_{10}}{\rho_{20}}
\end{gathered}
$$

The components of the total stress tensor can be now written as

$$
\begin{aligned}
t^{i i}=-p+T^{i i}, T^{i i} & =S^{i i}+\pi^{i i}, \text { no sum on } i \\
t^{i j} & =0, i \neq j .
\end{aligned}
$$

Finally, the components of the diffusive body force are given by

$$
b^{1}=-\frac{\mathrm{d}}{\mathrm{d} r}\left(\frac{\rho_{1}}{\rho_{10}}\right)+B, b^{2}=b^{3}=0,
$$

where

$$
B=-\rho_{10} K \frac{\mathrm{d}}{\mathrm{d} r}\left(\frac{\rho_{2}}{\rho_{20}}\right)-\rho_{10} K\left(\frac{\rho_{20}}{\rho_{10}}\right) \frac{\rho_{2}}{\rho_{20}} \frac{\mathrm{d}}{\mathrm{d} r}\left(\lambda_{r}^{2}+2 \lambda_{\theta}^{2}\right)-\alpha \frac{\rho_{1}}{\rho_{10}} \frac{\rho_{2}}{\rho_{20}} V
$$

We would like to eliminate the indeterminate scalar $p$ from eqns $(37)_{1,2}$ and $(39)_{1}$. Let eqn (35) be rewritten in the following form

$$
\frac{a}{h}\left(p_{1}-p_{2}\right)=2\left(t_{m}^{22}-t_{m}^{11}\right)+2 t_{m}^{11}
$$

Note that the first term on the r.h.s. is independent of the indeterminate scalar $p$. We next obtain an estimate for $t_{m}^{11}$ by integrating eqn (27) from $r_{i}$ to $a$,

$$
t^{11}(a)=t^{11}\left(r_{i}\right)-\int_{a-(h / 2)}^{a} \frac{2}{r}\left(t^{11}-t^{22}\right) \mathrm{d} r .
$$

It follows from eqns (35), (42), and the Mean Value Theorem, that eqn (41) can be rewritten as

$$
\frac{a}{h}\left(p_{1}-p_{2}\right)=2\left(t_{m}^{22}-t_{m}^{\prime \prime}\right)-2\left(1-\frac{h}{2 a}\right)^{-2} p_{1}+\left.2 \frac{h}{a}\left[\frac{a\left(t^{22}-t^{\prime \prime}\right)}{r}\right]\right|_{Q}
$$


In the above equation, the subscript $Q$ denotes that $a\left(t^{22}-t^{11}\right) / r$ is evaluated at some point $Q$ which lies between $r_{i}$ and $a$. Observe in eqn (43) that the last term on the r.h.s. is of order $h / a$ compared to the first term. The l.h.s. is of order $(a / h) p_{1}$, while the second term on the r.h.s. is of order $p_{1}$. Since $a / h \gg 1$, it follows that the first term on the r.h.s. is of order $a / h$, and the last two terms can be neglected in comparison. Hence, by virtue of eqns (37)-(39), (15) and (16), eqn (43) can be approximated by

$$
\begin{aligned}
\frac{a}{h}\left(p_{1}-p_{2}\right) & =2\left(t_{m}^{22}-t_{m}^{11}\right), \\
& -4 \rho_{10} K \rho_{m}\left(\lambda_{m}^{2}-\frac{1}{c^{2}}\right) .
\end{aligned}
$$

Next, we consider eqn (32) for the partial stresses for the solid. We need to develop an expression for $\hat{\sigma}_{*}^{11}$ and eliminate the scalar $p$ which appears in the expressions for $\sigma_{m}^{22}$ and $b_{m}$.

From eqn (39) $)_{1},-p=t^{11}-T^{11}$. Substituting into the expression for $\sigma^{11}$ given by eqn (37), and then using the definition in eqn (28), we obtain

$$
\hat{\sigma}^{\prime \prime}=\frac{\rho_{1}}{\rho_{10}} t^{11} \frac{r^{2}}{a^{2}}+\left(S^{11}-\frac{\rho_{1}}{\rho_{10}} T^{11}\right) \frac{r^{2}}{a^{2}}
$$

Next, using eqns (30) and (34), the following expression is obtained for the 1.h.s. of eqn (32),

$$
a \hat{\sigma}_{*}^{11}=\frac{a}{h}\left(p_{1}-p_{2}\right) \rho_{1 m}-\frac{a}{2} \rho_{1 *}\left(p_{1}+p_{2}\right)+a \hat{S}_{*}+O(h),
$$

where

$$
\hat{S}_{*}=\left.\frac{\mathrm{d}}{\mathrm{d} r}\left[\left(S^{11}-\frac{\rho_{1}}{\rho_{10}} T^{11}\right) \frac{r^{2}}{a^{2}}\right]\right|_{r=a}
$$

For the particular choice of the HElmholtz free energy function $A$ leading to eqns (38) $)_{1-3}$, $a \hat{S}_{*}^{11}$ is found to be

$$
a \hat{S}_{*}=\rho_{10} K\left[4 a c_{*} \frac{\rho_{m} \rho_{2 m}}{c}+\left(\frac{2}{c^{2}}-1\right)\left(2 \rho_{m} \rho_{2 m}+\rho_{m} \rho_{2 *} a+\rho_{2 m} \rho_{*} a\right)\right]
$$

By eqns (37) and (39), the r.h.s. of eqn (32) can be written as

$$
2 \sigma_{m}^{22}+a b_{m}=2 t_{m}^{11} \rho_{1 m}+a t_{m}^{11} \rho_{1 *}+2\left(S_{m}^{22}-T_{m}^{11} \rho_{1 m}\right)+a\left(B_{m}-T_{m}^{11} \rho_{1 *}\right),
$$

where use has been made of eqn (19), and the relation $-p=t^{11}-T^{11}$ from eqn (39). From eqn (42) and the corresponding result obtained by integrating from $a$ to $r_{0}$,

$$
t_{m}^{11}=\frac{1}{2}\left(t^{11}\left(r_{i}\right)+t^{11}\left(r_{0}\right)\right)+\int_{a}^{r_{o}} \frac{2}{r}\left(t^{11}-t^{22}\right) \mathrm{d} r-\int_{r_{i}}^{a} \frac{2}{r}\left(t^{11}-t^{22}\right) \mathrm{d} r
$$

Using eqn (34), eqn (49) can be rewritten as

$$
\begin{aligned}
t_{m}^{11} & =-\frac{1}{2}\left(p_{1}+p_{2}\right)+\frac{h}{a}\left[\left(t^{11}-t^{22}\right)_{Q_{0}}-\left(t^{11}-t^{22}\right)_{Q_{1}}\right]+O(h), \\
& =-\frac{1}{2}\left(p_{1}+p_{2}\right)+O(h),
\end{aligned}
$$

where the subscript $Q_{0}$ (or $Q_{1}$ ) denotes that $\left(t^{11}-t^{22}\right)$ is evaluated at some point $Q_{0}$ (or $Q_{1}$ ) between $a$ and $r_{0}$ (or between $r_{i}$ and $a$ ). 
Substituting from eqns (46) 1,2 and (48) into eqn (32), we obtain

$\frac{a}{h}\left(p_{1}-p_{2}\right) \rho_{1 m}-\frac{a}{2}\left(p_{1}+p_{2}\right) \rho_{1 *}+a \hat{S}_{*}+O(h)=2 t_{m}^{11} \rho_{1 m}+a t_{m}^{11} \rho_{1 *}+2\left(S_{m}^{22}-T_{m}^{11} \rho_{1 m}\right)+a\left(B_{m}-T_{m}^{11}\right) \rho_{1 *}$.

The terms involving $t_{m}^{11}$ in eqn (51) can now be eliminated using eqn (41) and (50). Neglecting terms of $O(h)$, we obtain

$$
2\left(t_{m}^{22}-t_{m}^{11}\right) \rho_{1 m}+a \hat{S}_{*}=2\left(S_{m}^{22}-T_{m}^{11} \rho_{1 m}\right)+a\left(B_{m}-T_{m}^{11} \rho_{1 *}\right) .
$$

Note that by means of the term $a B_{m}$, eqn (52) involves kinematic and density quantities evaluated at the midsurface in addition to their gradients.

\section{SATURATION CONDITION}

In this section we discuss the difficulties which one encounters in providing a clear definition of the boundary conditions for the partial stresses. The problem arises due to the fact that the total stresses, and not the individual partial stresses are specified on the boundaries. We indicated earlier that this difficulty could be overcome by utilizing eqn (8), provided the mixture is in a saturated state. We elaborate on the rationale for the same, below.

Treloar [5] considers the swelling of a unit cube of crosslinked material when the forces are applied to its faces in such a manner that the cube is constrained to deform into a rectangular block of dimension $l_{1}$, and other two edges remaining constant. Under a homogeneous strain, based on a thermodynamic requirement which governs equilibrium, namely that the Helmholtz free energy in a small displacement from equilibrium be equal to the work done by the applied forces, he obtains the following relation:

$$
t_{1}=\left(\frac{R T}{V_{1}}\right)\left[\ln (1-v)+\nu+\chi \nu^{2}+\left(\frac{\rho V_{1}}{M_{c}}\right) \nu 1_{1}^{2}\right]
$$

where $t_{1}$ is the force on the face perpendicular to the direction of length change. A similar derivation when a unit cube is constrained to deform into a rectangular block by forces applied on its faces while it is immersed in a bath with pressure $p_{s}$ leads to eqn (8).

Let us suppose that the spherical membrane under consideration has reached a saturated equilibrium state. The membrane is not in a state of homogeneous strain. However, consider an infinitesimal element on the surface of the membrane which is in contact with the diffusant. It we had a unit cube immersed in a bath at constant pressure and deformed it to the same homogeneous state of strain as the infinitesimal surface element, then eqn (8) would hold. Thus, as a first approximation, we could assume that eqn (8) holds at the infinitesimal element on the surface. Such an approximation is clearly in the spirit of much of what is done in mechanics in order to reduce intractable problems to be amenable to analysis. Moreover, such an approach has yielded results which are both quantitatively and qualitatively responsible in earlier work [3]. This condition, in addition to the total traction specified on the boundary makes the problem determinate.

We shall now proceed to the appropriate form of eqn (8) for the membrane theory valid at both the inner and outer boundaries of the membrane. For the sake of brevity we outline the membrane theory approximation of eqn (8) which is valid at the inner boundary. A similar approximation can be derived for the outer boundary. It follows from definition of the total stress, eqns (37)-(39), that

$$
\frac{t^{11}+t^{22}+t^{33}}{3}+P_{s}=-p\left(r_{i}\right)+\rho K \frac{\rho_{2}}{\rho_{20}} \frac{\rho_{10}}{\rho_{1}}+\frac{2}{3} \rho K\left(\lambda_{r}^{2}+2 \lambda_{\theta}^{2}\right)+q_{1}
$$

where the hydrostatic pressure of the fluid adjacent to the inner boundary is $q_{1}$. (Note from eqn (34) $)_{2}$ that $\left.q_{1}=p_{1}\left(a^{2} / r_{i}^{2}\right)\right)$. The following expression for $p\left(r_{i}\right)$ can be obtained from eqns $(34)_{2}$, 
(37)-(39)

$$
p\left(r_{i}\right)=q_{i}+2 \rho K \lambda_{s}^{2}+\rho K \frac{\rho_{2}}{\rho_{20}} \frac{\rho_{10}}{\rho_{1}}
$$

Substituting (53) and (54) into the Flory-Huggins eqn (8) onc obtains

$$
\frac{4}{3} \rho K \frac{V_{1}}{R T}\left(\lambda_{\theta}^{2}-\lambda_{r}^{2}\right)=\ln (1-\nu)+\nu+\chi^{2}+\rho_{10} \frac{V_{1}}{M_{c}}\left\{\frac{\nu}{3}\left(\lambda_{r}^{2}+2 \lambda_{\theta}^{2}\right)-\frac{\nu}{2}\right\} .
$$

We now proceed to obtain the membrane approximation for the above equation. Observe that the volume fraction of the solid constituent at the inner surface is given by

$$
\nu\left(r_{i}\right)=\left[\frac{1}{\lambda_{r} \lambda_{\theta}^{2}}\right]_{r_{i}}=v_{m}-v_{*} \frac{h}{2}
$$

where

$$
\nu_{m}=\left(\lambda_{m}^{2} / c\right)^{-1}
$$

and

$$
\nu_{*}=-\left(\lambda_{m}^{2} / c\right)^{-2}\left[2 \lambda_{*} \lambda_{m} / c+\lambda_{m}^{z} c_{*}\right]
$$

It follows from equations $(15)_{2},(16),(56)$ and $(57)_{1.2}$ that the boundary condition for the inner and outer boundaries are equivalent to the following relations

$$
\frac{4}{3} \rho_{10} \frac{K V_{1}}{R T} \rho_{m}\left(\lambda_{m}^{2}-\frac{1}{c^{2}}\right)=\ln \left(1-\nu_{m}\right)+\nu_{m}+\chi \nu_{m}^{2}+\frac{\rho_{10} V_{1}}{M_{c}}\left[\frac{\nu_{m}}{3}\left(2 \lambda_{m}^{2}+\frac{1}{c^{2}}\right)-\frac{v_{m}}{2}\right]
$$

and

$$
\begin{aligned}
\frac{4}{3} \rho_{10} \frac{K V_{1}}{R T}\left\{\rho_{*}\left(\lambda_{m}^{2}-\frac{1}{c^{2}}\right)+2 \rho_{m}\left(\lambda_{m} \lambda_{*}-\frac{c_{*}}{c}\right)\right\} & =\nu_{*}\left(2 \chi \nu_{m}+1-\frac{1}{1-v_{m}}\right) \\
& +\frac{\rho_{10} V_{1}}{M_{c}}\left\{\frac{\nu_{*}}{3}\left(2 \lambda_{m}^{2}+\frac{1}{c^{2}}\right)+\frac{2 \nu_{m}}{3}\left(2 \lambda_{m} \lambda_{*}+\frac{c_{*}}{c}\right)-\frac{\nu_{*}}{2}\right\} .
\end{aligned}
$$

\section{SUMMARY OF VARIABLES AND EQUATIONS}

The membrane theory developed here involves 12 dependent variables, representing the values and radial gradients of the various physical quantities at the deformed middle surface. These variables are: (a) the radial and circumferential stretch ratios-3 variables, $a / A, c, c_{*}$; (b) the fluid, solid and total densities-6 variables, $\rho_{1 m}, \rho_{1 *}, \rho_{2 m}, \rho_{2 *}, \rho_{m}, \rho_{*} ;$ (c) the fluid radial velocity -2 variables, $V_{m}, V_{*}$; (d) the mass flux $F-1$ variable.

The 12 equations for these variables are given by: (a) conservation of mass for the solid-2 eqns $(20)_{1}$ and $(20)_{2}$; (b) conservation of mass for the fluid-2 eqns $(25)_{1}$ and $(25)_{2}$; (c) volume additivity constraint -2 eqns $(21)_{1}$ and $(21)_{2}$; (d) total density definition -2 eqns $(22)_{1}$ and (22) ; (e) the total stress equilibrium equation-1 eqn (44); (f) the partial stress-diffusive body force equilibrium equation -1 eqn (52); and (g) the saturation conditions- 2 eqns (58) and (59).

This system of equations can be reduced to a lower order for $\lambda_{m}, c, c_{*}$ and $F$. To achieve this, we define the dimensionless pressure difference

$$
P=\frac{A\left(p_{1}-p_{2}\right)}{4 h_{0} \rho_{10} K}
$$

Then, by eqns (15) 1.2 and (17), the total stress equilibrium eqn (44) can be written as

$$
P=\frac{\rho_{m}}{\lambda_{m} c}\left(\lambda_{m}^{2}-\frac{1}{c^{2}}\right)
$$


Next, substitute from eqns (44), (47), (38) $)_{1,2}$ and (40) $)_{4}$ into eqn (52). Let $V_{m}$ be eliminated in terms of $F$ by using eqn (25). On setting $a=\lambda_{m} A$ and dividing through by $\rho_{10} K$, eqn (52) becomes

$$
\begin{aligned}
4 \rho_{m} \rho_{1 m} & \left(\lambda_{m}^{2}-\frac{1}{c^{2}}\right)+\left[4 \lambda_{m} \rho_{m} \rho_{2 m} \frac{c_{*} A}{c}+\left(\frac{2}{c^{2}}-1\right)\left(2 \rho_{m} \rho_{2 m}+\rho_{m} \lambda_{m} \rho_{2 *} A+\lambda_{m} \rho_{2} m \rho_{*} A\right)\right] \\
= & 4 \rho_{m}\left(\lambda_{m}^{2}-\frac{\rho_{1 m}}{c^{2}}\right)+\lambda_{m}\left[-\rho_{2 *} A-\rho_{2 m} \frac{\rho_{20}}{\rho_{10}}\left(4 \lambda_{m} \lambda_{*} A+\frac{2 c_{*} A}{c}\right)-2 \rho_{m} \frac{\rho_{1 *} A}{c^{2}}-\hat{F} \frac{\rho_{1 m}}{\lambda_{m}^{2}}\right],
\end{aligned}
$$

where $\hat{F}$ is the dimensionless mass flux defined by the relation

$$
\hat{F}=\frac{\alpha F}{A \rho_{10} \rho_{20} K}
$$

Now observe that $\rho_{m}, \rho_{1 m}$ and $\rho_{2 m}$ can be expressed in terms of $\lambda_{m}$ and $c$ by eqns (20), $(21)_{1}$ and (22) $)_{1}$. Since by eqn $(15)_{1,2}$

$$
\lambda_{*} A=\left(1-\lambda_{m} c\right),
$$

$\rho_{*} A, \rho_{1 *} A$ and $\rho_{2 *} A$ can be expressed in terms of $\lambda_{m}, c$ and $c_{*} A$ by eqns $(20)_{2},(21)_{2}$ and $(22)_{2}$. Thus, eqn (61) is of the form

$$
P=\phi_{1}\left(\lambda_{m}, c\right),
$$

while eqn (62) is of the form

$$
\phi_{2}\left(\lambda_{m}, c, c_{*} A, \hat{F}\right)=0
$$

In view of the above remarks and definitions (57) 1.2 , the saturation eqns (58) and (59) respectively can be seen to be of the form,

$$
\phi_{3}\left(\lambda_{m}, c\right)=0, \phi_{4}\left(\lambda_{m}, c, c_{*} A\right) \div 0
$$

Suppose the geometric properties of the undeformed membrane, the relevant properties of the solid and fluid constituents, and the pressure difference $p_{1}-p_{2}$, are given. Then $\lambda_{m}$ and $c$ are first found from eqns (65) and (67) $)_{1}$. Next $c_{*} A$ is found from eqn $(67)_{2}$ and then the mass flux $\hat{F}$ is found from eqn (66).

\section{NUMERICAL EXAMPLE}

Calculations were carried out using the same data as was used in previous work [4], in which a rubber-toluene mixture was considered. For the rubber, the constant $K$ was computed using $T=303.16^{\circ}, M=919 \mathrm{~g} / \mathrm{mole}, R=8.317 \times 10^{7}$ dyne-cm $/ \mathrm{mole}-{ }^{\circ} \mathrm{K}$, and density $\rho_{10}=0.862 \mathrm{~g} / \mathrm{cm}^{3}$. For toluene, $V_{1}=106 \mathrm{~cm}^{3} /$ mole and the molecular weight is $92.13 \mathrm{~g} / \mathrm{mole}$, giving $\rho_{20}=$ $0.869 \mathrm{~g} /$ mole. The value of the constant $\chi$ in the saturation eqn (8) was taken as 0.425 .

According to the results presented in [3], the gradient of $\lambda_{r}$ or $\lambda_{\theta}$ with respect to $R / R_{i}$ is negative and has a magnitude of about 0.12 , when $\hat{F}=150$. In order to compare this with the results in the present example, first note that the following relations are readily established.

$$
\frac{\mathrm{d} \lambda_{\theta}}{\mathrm{d}\left(R / R_{i}\right)} \doteq \frac{\lambda_{*} A}{c}, \frac{\mathrm{d} \lambda_{r}}{\mathrm{~d}\left(R / R_{i}\right)}=\frac{c_{*} A}{c}
$$

It was found that $\lambda_{*} A / c \approx-0.104$ and $c^{*} A / c \approx-0.107$, which is in very good agreement with the results in [4]. The terms in eqn (62) involving $\rho_{*} A, \rho_{1 *} A, \rho_{2 *} A$ were very small, whereas the term involving $\lambda_{*} A$ and $c_{*} A$ was $36 \%$ of the term involving the dimensionless flux $\hat{F}$. It is not clear whether the smallness of the density gradients is due to the data used in the 
calculations, or is a natural result of the membrane theory. In the latter case, one could neglect these terms and simplify the equations. However, our results indicate that the terms in $\lambda_{*} A$ and $c_{*} A$ cannot be neglected.

Each solution of the system of eqns (65)-(67) represents an equilibrium state of the spherical membrane, in which the material particles have swollen with fluid to a fixed saturated state, and through which there is steady flow of fluid. When the dimensionless pressure $P$ equals zero, it follows from eqn (65) (i.e. eqn 61), that $\lambda_{m}=c^{-1}$, and that each material element has undergone unconstrained swelling. The membrane radius has increased, with $\lambda_{m}=1.78$. The midsurface stretch ratiọs $\lambda_{m}$ and $c^{-1}$ increase with $P$ until $\lambda_{m} \approx 2.5$, then $P$ begins to decrease with increasing $\lambda_{m}$. This interesting result is the extension to mixture theory of the well known lack of monotonicity in the relation between pressure and radius in the inflation of a neo-Hookean elastic spherical membrane. The gradients $\lambda_{*}$ and $c_{*}$ increase with $\lambda_{m}$ until $\lambda_{m} \approx 2.3$, when they decrease. The flux increases monotonically with $\lambda_{m}$. Plots of $\lambda_{m}$ vs $c^{-1}, P$ and $F$ are shown in Fig. 1 and a plot $P$ vs $\hat{F}$ is shown in Fig. 2.

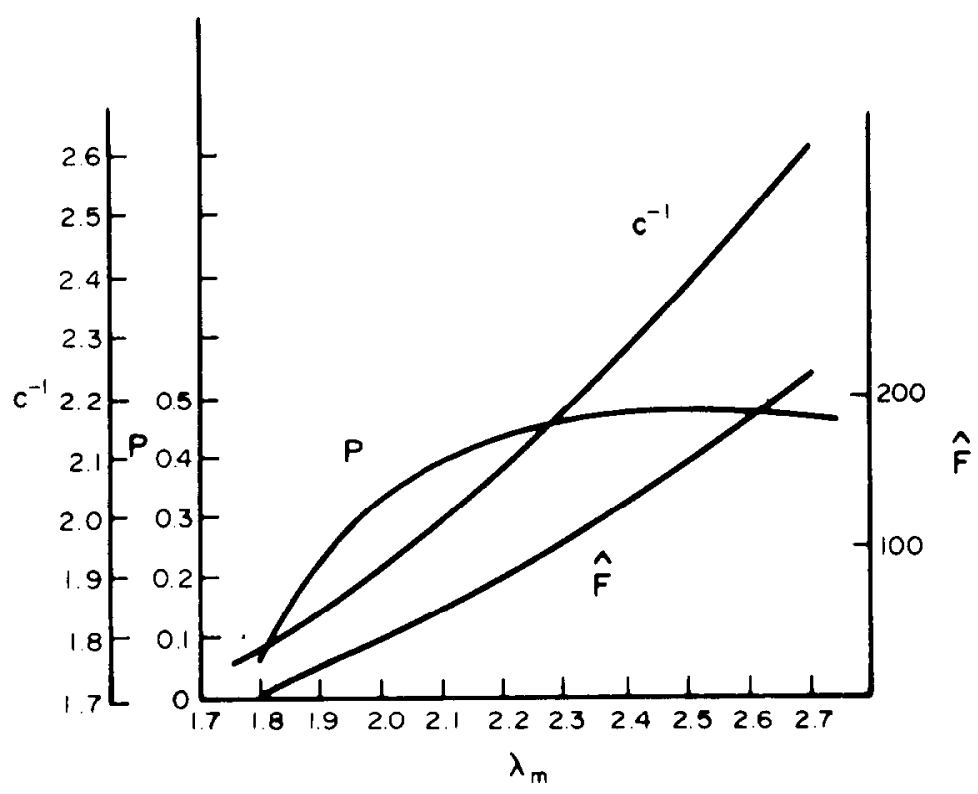

Fig. 1. Variation of dimensionless pressure difference $P$, dimensionless flux $\hat{F}$ and midsurface radial stretch ratio with the midsurface circumferential stretch ratio.

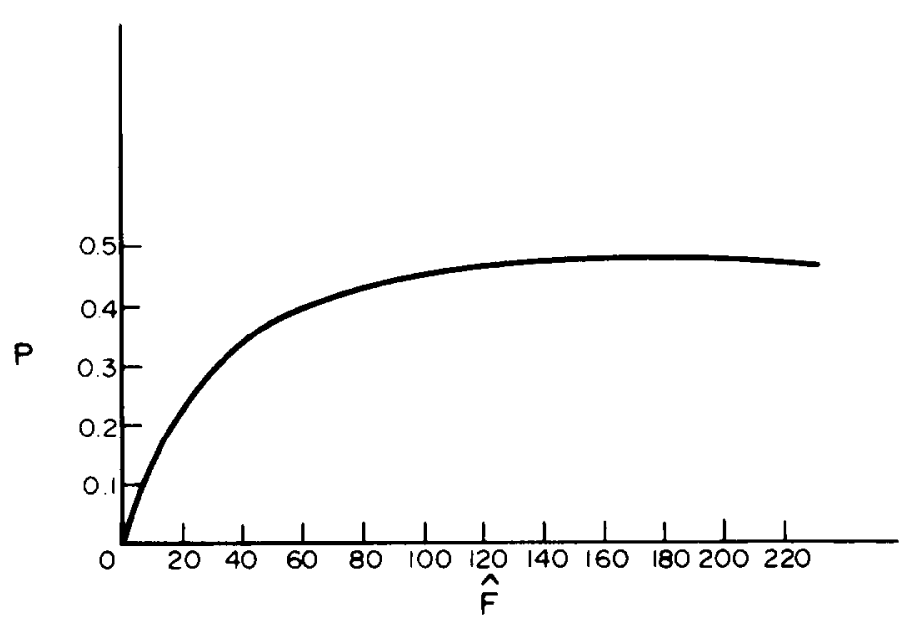

Fig. 2. Variation of dimensionless pressure difference $P$ with dimensionless flux $\hat{F}$. 
Acknowledgements-We wish to thank Mr. Mukesh Gandhi for his computational assistance. We also wish to thank the Macromolecular Research Center of The University of Michigan for its support of $\mathrm{K}$. R. Rajoagopal during the course of this research.

\section{REFERENCES}

[1] A. E. GREEN and J. E. ADKINS, Large Elastic Deformations. Clarendon Press, Oxford (1960).

[2] A. S. WINEMAN, Trans. Soc. Rheol. 20, 203-225 (1976).

[3] J. J-J. SHI, Application of a theory of a Newtonian fluid and on isotropic non-linear elastic solid to diffusion problems. Doctoral Dissertation, The University of Michigan (1973).

[4] J. J-J. SHI, K. R. RAJAGOPAL and A. S. WINEMAN, Applications of the theory of interacting continua to the diffusion of a fluid through a non-linear elastic media. Int. J. Engng Sci. 19, 871-889 (1981).

[5] L. R. TRELOAR, The Physics of Rubber Elasticity. Clarendon Press, Oxford (1975).

[6] $\Lambda$. E. GREEN and P. M. NAGHDI, Q. J. Mech. Appl. Math. 22, 427-438 (1969).

[7] N. MILLS, Int. J. Engng Sci. 4, 97-112 (1966).

[8] J. E. ADKINS, Non-linear diffusion-III. Diffusion through isotropic highly elastic solids. Phil. Trans. Roy. Soc. A256, $301-316(1964)$. 